\title{
The Effect of Active and Non-Active Follow-Up of Patients on the Recurrence of Ischemic Stroke
}

\author{
Davood Kashipazha, ${ }^{1}$ Ahmad Ghomifar, ${ }^{2,}{ }^{*}$ and Farzaneh Karimian ${ }^{2}$ \\ ${ }^{1}$ Assistant Professor of Department of Neurology, School of Medicine, Ahvaz Jundishapur University of Medical Sciences, Ahvaz, IR Iran \\ ${ }^{2}$ Neurology Resident of Department of Neurology, School of Medicine, Ahvaz Jundishapur University of Medical Sciences, Ahvaz, IR Iran \\ "Corresponding author: Ahmad Ghomifar, Golestan Hospital, Farvardin Street, Ahvaz, Iran. Tel: +98-6133743012, Fax: +98-6133743012, E-mail: aghomifar@yahoo.com
}

Received 2017 May 27; Revised 2017 June 16; Accepted 2017 September 16.

\begin{abstract}
Background: Stroke is the third cause of death in the US. Due to its associated mortality and morbidity, it is a very important disease, and recurrent stroke increases the risk of both conditions. Atherosclerotic risk factors are the most common, and yet modifiable, risk factors for stroke. Therefore, stroke prevention should be focused on the treatment of these underlying factors of the disease. Despite the same medical treatments for this disease in various communities, the discernable difference in recurrent stroke in different studies (ranging from $3 \%$ to $23.2 \%$ ) suggests this difference can be attributed to the lack of appropriate control of modifiable risk factors for this condition in communities with higher incidence of stroke. Hence, the present study aimed at testing this hypothesis.

Methods: In this randomized clinical trial study, 76 patients with ischemic stroke were assigned to two groups of active follow-up and non-active follow-up of risk factors. The subjects in the two groups were studied and compared with respect to control of risk factors and recurrent ischemic stroke at the beginning and at the end of the research.

Results: The rates of recurrent stroke within 6 months from the beginning of the study in the active and non-active follow-up groups were $2.8 \%$ and $13.8 \%$, respectively. In addition, the chance of recurrent stroke in the non-active follow-up group was 5 times more than that in the active follow-up group ( $\mathrm{OR}=5, \mathrm{CI}=95 \%)$.

Conclusions: Active follow-up of patients after the first stroke leads to a better control of ischemic stroke risk factors and reduces its recurrence rate.
\end{abstract}

Keywords: Stroke, Stroke Rehabilitation, Atherosclerosis, Follow-Up Studies

\section{Background}

Stroke is among the five leading causes of death in most countries (1). Moreover, since this condition causes cognitive, emotional, and physical disabilities and it is responsible for 3.6\% of the total disability-adjusted life years (DALYs), it is considered one of the 10 causes of disability in all countries (2). Among all the neurologic diseases of adult life, stroke ranks first in frequency and importance (3). The common mode of expression of stroke is a relatively sudden occurrence of a focal neurologic deficit (4). Strokes are broadly categorized as ischemic or hemorrhagic. Ischemic stroke is due to occlusion of a cerebral blood vessel causing cerebral infarction (5). Based on the underlying causes, ischemic stroke is divided into 5 major categories of large artery atherosclerosis (15\% - 40\%), cardioembolic stroke (15\% - 30\%), small artery occlusion or lacunar stroke $(15 \%$ - 30\%), cryptogenic stroke (up to $40 \%$ ), and other causes (5\%) (6). The treatment of stroke may be divided into three parts: management in the acute phase by measures to restore the circulation and arrest the pathologic process, physical therapy and rehabilitation, and measures to prevent further strokes and progression of vascular disease (7). It is now a major goal of general medicine to reduce the incidence of stroke in the general population by the control of modifiable risk factors ("primary prevention") (8). In addition to reduction of known risk factors such as hypertension, smoking, and glucose control in diabetics (9), the widespread use of cholesterol-lowering statin medications and antiplatelet drugs has shown in some studies to reduce the primary incidence of and recurrence of stroke (10). Atherosclerotic risk factors are the most common, and yet the most modifiable, risk factors for stroke. Atherosclerotic risk factors include high blood pressure, diabetes mellitus, smoking, and dyslipidemia (11). The discernable difference in recurrent stroke in different studies (ranging from $3 \%$ to $23.2 \%$ ) $(12,13)$, despite the use of the same medical treatments for this disease in various com- 
munities, suggests the hypothesis that this difference can be attributed to the lack of appropriate control of modifiable risk factors for this condition in communities where the incidence of stroke is higher.

\section{Objectives}

The present study aimed at finding out whether strict control of atherosclerotic risk factors after the first stroke could reduce the chance of recurrent stroke.

\section{Methods}

\subsection{Patients}

In this randomized clinical trial study, 86 patients, diagnosed with ischemic stroke by neurologists and hospitalized in the neurology ward of Golestan hospital in Ahwaz, entered the study. The sample size according to previous studies (14) was calculated for each group as 36 patients. However, to compensate for sample loss, 43 patients were allocated to each group. The inclusion criteria were no history of heart valve problems, cardiac arrhythmia, congestive heart failure (ejection fraction $<35 \%$ ), stenosis of more than $70 \%$ in the doppler ultrasound of the cervical vessels, and a national institutes of health stroke scale (NIHSS) of below 22 (15). Moreover, the exclusion criteria included inability of patients to participate in clinical assessments due to a medical condition.

\subsection{Design}

The subjects were assigned to two groups of active follow-up and non-active follow-up of risk factors using the block randomization method. The same medical procedures were conducted for both the groups during their hospitalization. The only difference between the two groups was in terms of the day of discharge; subjects in the non-active follow-up group only received oral recommendations for periodic visits to the specialized clinic of Golestan hospital (every two weeks for one month and then once every month for 6 months after discharge), while in the active follow-up group, in addition to oral recommendations, regular visits of patients to a physician at specified dates were reminded and followed up. At each visit to the physician, blood pressure, blood glucose, and low-density lipoprotein (LDL) of patients were measured and appropriate drugs were prescribed. Subjects in the active follow-up group at each visit and those in the nonactive follow-up group at the end of the 6-month period were again questioned and examined in relation to recurrent stroke. Of the 86 patients who initially entered the study, 5 were excluded from the active follow-up group because they did not continue their follow-up visits. In the non-active follow-up group, 4 subjects were also eliminated because they were inaccessible at the end of the 6-month period. In addition, one patient in this group died of heart attack during the study and therefore, excluded from the research. As a result, a total of 76 patients completed the study. The study CONSORT flow diagram is shown in Figure 1.

\subsection{Data Analysis}

Statistical analysis was conducted using SPSS software (version 20). The percentage for the nominal variables was calculated. Mean \pm standard deviation was calculated for each continuous variable. Odds ratio (OR) and exact poisson confidence intervals (CI) were calculated. For each group, prevalence of risk factors and recurrence of stroke were compared by paired-sample $t$ - test. $P$ value of less than 0.05 was considered statistically significant.

\subsection{Ethical Considerations}

This study was approved by the ethics committee of Ahvaz Jundishapur University of Medical Sciences (code: IR.AJUMS.REC.1394.22), and consent forms were obtained from all patients who participated in the research. The trial was registered at the Iranian registry of clinical trials (http://www.irct.ir) with the Irct ID: IRCT2015051212781N3.

\section{Results}

Of the patients in the present study, $64.8 \%$ were male and $35.2 \%$ were female. The mean age of the subjects was $65.9 \pm 11.53$ with a minimum and maximum of 41 and 90 , respectively. At the start of study, there was no significant difference between subjects of the two groups in terms of gender, age, stroke site, diabetes mellitus, smoking history, dyslipidemia, blood pressure, and NIHSS (Table 1). At the end of the study, recurrent stroke was observed in 5 subjects of the non-active follow-up group (13.8\%) and only in one subject of the active follow-up group (2.8\%) (Table 2). The $P$ value resulting from the comparison of recurrent stroke rate between the two groups was not significant at the level of 0.05. However, due to the closeness of the $P$ value to the significance level $(\mathrm{P}=0.089)$ and since the odds ratio of recurrent stroke in the non-active follow-up group was 5 times more than that in the active follow-up group $(\mathrm{OR}=5, \mathrm{CI}=95 \%)$, it can be concluded that active follow-up of patients is effective in reducing the risk of recurrent ischemic stroke. 


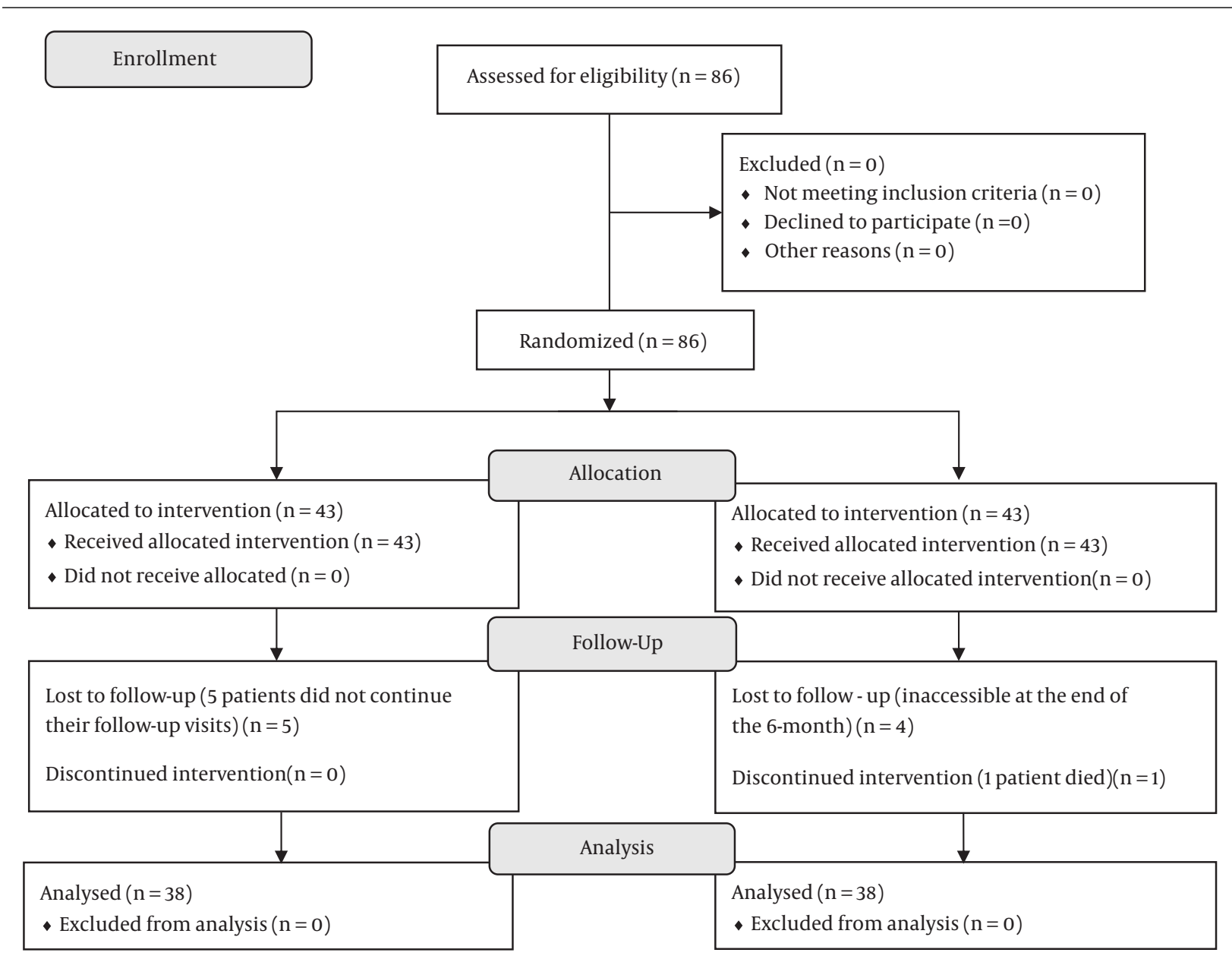

Figure 1. Study CONSORT Flow Diagram

\section{Discussion}

Stroke is one of the leading causes of mortality and morbidity in different communities. Therefore, stroke prevention is one of the main goals of health systems. Recurrent stroke increases the risk of mortality and morbidity caused by stroke. It seems the control of modifiable risk factors for stroke can reduce the odds of recurrent stroke in patients previously experiencing a stroke. Shamsaei et al., in a study on 590 patients, showed that high blood pressure was a risk factor for recurrent stroke(16). Mazdeh et al. also conducted a study on 5413 subjects and concluded that the major risk factors for stroke recurrence included high blood pressure, diabetes mellitus, smoking, and dyslipidemia (17). Therefore, in the present study, high blood pressure, diabetes mellitus, dyslipidemia, and smoking were identified as the most important risk factors for recurrent ischemic stroke. In this study, the rate of recurrent stroke within 6 months from the beginning of the study was $2.7 \%$ in the active follow-up group and $13.8 \%$ in the non- active follow-up group. In addition, the odds ratio in the nonactive follow-up group was 5 times more than the active follow-up group.

The rate of recurrent stroke in the active follow-up group was close to the findings of Achterberg et al. (2013) in the Netherlands who reported that the risk of recurrent stroke after the first stroke of small artery and large artery was 3.3\% and $2.9 \%$ per year, respectively (12). These figures were also similar to the results of a meta-analysis carried out by Mohan et al. (2014) on 9115 patients in Europe and north American countries, finding that the cumulative risk of recurrent stroke (ischemic and embolic) was $3.1 \%$ within the first month and $11.1 \%$ within the first year (18). By contrast, the rate of recurrent stroke in the non- active follow-up group was consistent with the findings of Mazdeh in Hamedan, I.R.Iran, who reported that the risk of recurrent stroke in patients was $17.35 \%$ (17). This 
Table 1. Comparison of the Prevalence of Risk Factors Between Two Groups at the Start of the Study

\begin{tabular}{|c|c|c|c|c|c|c|c|c|c|}
\hline & \multicolumn{2}{|c|}{ HTN } & \multicolumn{2}{|c|}{ Dyslipidemia } & \multicolumn{2}{|c|}{ Smoking } & \multicolumn{2}{|c|}{ Diabetes Mellitus } & \multirow[t]{2}{*}{ NIHSS $^{a}$ Mean } \\
\hline & - & + & - & + & - & + & - & + & \\
\hline Active follow-up & $14(36.8 \%)$ & $24(63.2 \%)$ & $29(76.3 \%)$ & $9(23.7 \%)$ & $27(71.1 \%)$ & $11(28.9 \%)$ & $23(60.5 \%)$ & $15(39.5 \%)$ & 14.81 \\
\hline Non-active follow-up & $12(31.6 \%)$ & $26(68.4 \%)$ & $27(71.1 \%)$ & $11(28.9 \%)$ & $28(73.7 \%)$ & $10(26.3 \%)$ & $23(60.5 \%)$ & $15(39.5 \%)$ & 14.13 \\
\hline Pvalue & & 0.629 & & 0.602 & & 0.798 & & 1.000 & 0.405 \\
\hline
\end{tabular}

${ }^{a}$ National Institutes of Health Stroke Scale.

Table 2. Comparison of Recurrent Stroke Rate Between Two Groups at the End of the Study

\begin{tabular}{|c|c|c|c|c|}
\hline & \multicolumn{2}{|c|}{ Group } & \multirow[b]{2}{*}{ P Value } & \multirow[b]{2}{*}{ Odds Ratio } \\
\hline & Non-Active & Active & & \\
\hline With recurrent stroke & $5(13.2 \%)$ & $1(2.7 \%)$ & & \\
\hline Without recurrent stroke & $33(86.8 \%)$ & $37(97.3 \%)$ & 0.089 & $5(\mathrm{CI}=95 \%)$ \\
\hline
\end{tabular}

was also similar to the rate of recurrent stroke (23.2\%) reported by Ghandehari (2013) in Mashhad, I.R.Iran(13). All in all, considering the difference between the active and nonactive follow-up groups in terms of recurrent stroke rate (that was five times higher in the non-active group than in the active group), the similarity of recurrent stroke rate obtained in non-active follow-up group on this research with the findings in previous studies in Iran, and also the similarity of this rate in the active follow-up group with that in more advanced societies where the risk factors are controlled accurately and actively, it can be concluded that active follow-up of patients with stroke and strict control of risk factors can lead to reduced rate of recurrent stroke and consequently, the attributed rates of mortality and morbidity.

\subsection{Limitations}

Because of the small number of patients with recurrent stroke in this study, the relationship between risk factors and recurrent stroke was not specified. It is recommended to conduct a similar study with a larger sample size to find the relationship between risk factors and the incidence of recurrent stroke.

\subsection{Conclusion}

According to the results of the present study, active follow-up of patients after the first stroke and strict control of ischemic stroke risk factors can reduce the rate of recurrent stroke.

\section{Acknowledgments}

We would like to thank Dr. Shahram Rafi for comments that greatly improved the manuscript.

\section{Footnotes}

Conflict of Interest Statement: The authors have no conflict of interest.

Trial Registration: The trial was registered at the Iranian Registry of Clinical Trials (http://www.irct.ir) with the Irct ID: IRCT2015051212781N3

\section{References}

1. Torfi KA, Birgani AG. Assessment of quality of life and mental health in patients with brain stroke in Tehran Shariati Hospital. JJCDC. 2012;1(1):9-1.

2. Ropper AH, Samuels MA, Klein JP. Adams and Victor's principles of neurology. 10 ed. ; 2014.

3. Bandasak R, Narksawat K, Tangkanakul C, Chinvarun Y, Siri S. Association between hypertension and stroke among young Thai adults in Bangkok, Thailand. Southeast Asian J Trop Med Public Health. 2011;42(5):1241-8. [PubMed: 22299451].

4. De Lima LG, Soares BG, Saconato H, Atallah AN, da Silva EM. Betablockers for preventing stroke recurrence. Cochrane Database Syst Rev. 2013(5):CD007890. doi: 10.1002/14651858.CD007890.pub2. [PubMed: 23728669].

5. Katz BS, Flemming KD. Antithrombotic selection and risk factor management in ischemic stroke and transient ischemic attack. Neurosurg Focus. 2014;36(1):E10. doi: 10.3171/2013.10.FOCUS13402. [PubMed: 24380476].

6. Louis EA, Mayer SP, Rowland L. Merritt's neurology.13 ed.; 2016. p. 269

7. Kono Y, Yamada S, Yamaguchi J, Hagiwara Y, Iritani N, Ishida S, et al. Secondary prevention of new vascular events with lifestyle intervention in patients with noncardioembolic mild ischemic stroke: a single-center randomized controlled trial. Cerebrovasc Dis. 2013;36(2):88-97. doi: 10.1159/000352052. [PubMed: 24029303].

8. Niewada M, Czlonkowska A. Prevention of ischemic stroke in clinical practice: a role of internists and general practitioners. Pol Arch Med Wewn. 2014;124(10):540-8. [PubMed: 25369511].

9. Purroy F, Jimenez Caballero PE, Gorospe A, Torres MJ, Alvarez-Sabin J, Santamarina E, et al. Recurrent transient ischaemic attack and early risk of stroke: data from the PROMAPA study. J Neurol Neurosurg Psychiatry. 2013;84(6):596-603. doi: 10.1136/jnnp-2012-304005. [PubMed: 23345284]. 
10. Yoon Y, Lee DH, Kang DW, Kwon SU, Suh DC, Bang OY, et al. Stroke recurrence patterns are predicted by the subtypes and mechanisms of the past, non-cardiogenic stroke. Eur J Neurol. 2013;20(6):928-34. doi: 10.1111/ene.12101. [PubMed: 23398300].

11. Chen C, Zhao Y, Zhang J, Wang H, Wang X, Ma X. Analysis of multiple risk factors for the recurrence of nondisabling stroke. J National Med Asso. 2012;104(7-8):331-5.

12. Achterberg S, Pruissen DM, Kappelle LJ, Algra A, Smart Study Group . Risk of vascular events after nondisabling small and large vessel cerebral ischemia. Cerebrovasc Dis. 2013;36(3):190-5. doi: 10.1159/000353675. [PubMed: 24135529].

13. Ghandehari K, Khajedaluei MR, Yazdankhah Z, Ghandehari K. Risk factors of short-term stroke recurrence in patients with minor ischemic cerebrovascular events. ARYA Atheroscler. 2013;9(2):119-27. [PubMed 23690811].

14. Cheng EM, Jolly D, Jones LA, Cohen SN. Modest improvement in risk factor control after admission for a stroke or transient ischemic attack. J Stroke Cerebrovasc Dis. 2005;14(4):174-8. doi: 10.1016/j.jstrokecerebrovasdis.2005.03.003. [PubMed: 17904021].

15. Cheung CM, Tsoi TH, Hon SF, Au-Yeung M, Shiu KL, Lee CN, et al. Using the National Institutes of Health Stroke Scale (NIHSS) to predict the mortality and outcome of patients with intracerebral haemorrhage. Hong Kong Med J. 2008;14(5):367-70. [PubMed: 18840907].

16. Shamsaee MH, Tavangar A, Chitsaz A, Akbari M, Saadatnia M. Epidemiologic evaluation and seasonal variations of stroke risk factors among patients with first and recurrent stroke.J Isfahan Med School. 2012;29.

17. Mazdeh M, Torabian S, Zafari P. Recurrent stroke in hospitalized patients, in Farshchian hospital of Hamadan, Iran. Hamedan J Med Sci. 2010;16(4).

18. Mohan KM, Wolfe CD, Rudd AG, Heuschmann PU, Kolominsky-Rabas PL, Grieve AP. Risk and cumulative risk of stroke recurrence: a systematic review and meta-analysis. Stroke. 2011;42(5):1489-94. doi: 10.1161/STROKEAHA.110.602615. [PubMed: 21454819]. 